\title{
La CSS y triangular frente a los retos de la Agenda de ODS: Oportunidades y desafíos de la transferencia de tecnología como herramienta para el desarrollo sostenible y la superación de desigualdades
}

\section{Lorena Lamas'}

Resumen: El trabajo pretende realizar un aporte a las reflexiones sobre la necesidad de incentivar la cooperación y transferencia tecnológica, como herramienta de gran relevancia para la consecución de los Objetivos de Desarrollo Sostenible (ODS) y disminución de las desigualdades del Sur. Si bien las acciones en este sentido no son nuevas, los desafíos continúan existiendo y han ido transformándose en su forma y complejidad; requiriendo, por tanto, de nuevos abordajes y respuestas innovadoras.

En función de ello, el presente trabajo se cuestiona acerca de las posibles acciones a ser llevadas adelante por los países, en particular los latinoamericanos -por ser la región más desigual del mundo-, para enfrentar estos desafíos.

A partir de dicho cuestionamiento, se realiza un breve análisis con el objetivo de identificar algunos de los desafíos y oportunidades actuales para el cumplimiento de los ODS y la disminución de las desigualdades del Sur.

Del referido análisis, se esbozan algunas propuestas que ponen su foco en la utilización de las nuevas tecnologías de carácter exponencial como una herramienta clave e innovadora para alcanzar estos objetivos, en función de su capacidad de aumentar los impactos a costos reducidos; al tiempo que permiten disminuir la brecha tecnológica Norte-Sur, la cual retrasa el desarrollo global y, por tanto, la posibilidad de cumplir los ODS.

\footnotetext{
' Lorena Lamas, Doctora en Relaciones Internacionales (Universidad Nacional de Rosario). Profesora de Derecho Internacional y Cooperación internacional para el desarrollo sustentable. Investigadora de CID, CSS, APE, ODS, innovación y reducción de desigualdades.
} 
La hipótesis que orientó el trabajo se relaciona con la necesidad de dar respuestas innovadoras e impulsar diferentes asociaciones y proyectos de cooperación que involucren diversas modalidades y actores del Sistema Internacional en la consecución de los ODS y disminución de las desigualdades.

Finalmente, a partir del análisis realizado, el trabajo propone la implementación de un tipo de cooperación internacional horizontal basada en la utilización de tecnologías exponenciales para superar las 169 metas establecidas en los 17 ODS y disminuir las desigualdades, a la cual se propone denominar: Cooperación Exponencial (CoEx).

Palabras clave: Objetivos de Desarrollo Sostenible (ODS), tecnologías exponenciales, Cooperación Exponencial (CoEx).

Fecha de recepción: 4 de febrero de 2019.

Fecha de admisión definitiva: I I de marzo de 2020.

\section{South-South and Triangular Cooperation towards the SDGs Agenda: Opportunities and challenges of technology transfer as a tool for sustainable development and overcoming inequalities}

Abstract: The paper aims to contribute on the reflections about the need to encourage cooperation and technology transfer, as a relevant tool for achieving the Sustainable Development Goals (SDGs) and reducing South inequalities. Although the actions in this sense are not new, the challenges continue to exist and have been transformed in their form and complexity; requiring, therefore, new approaches and innovative answers.

Based on this, the work questioning about the possible actions to be taken by the countries, in particular the Latin American ones-because it is the most unequal region in the world-, to face these challenges.
La Coopération Sud-Sud et Triangulaire face aux défis de l'Agenda ODD: Opportunités et défis du transfert de technologie en tant qu'outil de développement durable et de lutte contre les inégalités

Résumé: Ce travail vise à apporter une contribution aux réflexions sur la nécessité $d$ 'encourager la coopération et le transfert de technologie, en tant qu' outil d'une grande pertinence pour la réalisation des objectifs de développement durable (ODD) et la réduction des inégalités du sud. Bien que les actions en ce sens ne soient pas nouvelles, les défis continuent $d$ 'exister et se sont transformés dans leur forme et leur complexité; ils exigent donc de nouvelles approches et des réponses innovantes.

En conséquence, ce travail pose des questions sur les actions possibles à entreprendre par les pays, en particulier les pays d'Amérique latine - en tant que région la plus inégale 
Based on this questioning, a brief analysis is carried out with the objective of identify some of the current challenges and opportunities for compliance with the SDGs and the reduction of inequalities in the South.

Based on the aforementioned analysis, some proposals are outlined focusing on the use of new exponential technologies as a key and innovative tool to achieve these objectives, based on their capacity to increase impacts at reduced costs; allowing at the same time, the reduction of the North-South technological gap, which delays global development and, therefore, the possibility of the SDGs overcoming.

The hypothesis that guided the work is related to the need of provide innovative answers and promote different partnerships and cooperation projects that involve different modalities and actors of the International System in achieving the SDGs and reducing inequalities.

Finally, based on the analysis, the work proposes the implementation of a horizontal international cooperation based on the use of exponential technologies to overcome the SDGs and reduce inequalities, which it is proposed to be named: Exponential Cooperation (ExCo).

Key words: Sustainable Development Goals (SDGs), exponential technologies, Exponential Cooperation (ExCo). du monde - pour faire face à ces défis. Sur la base de ce questionnement, une brève analyse est effectuée dans le but d'identifier certains des défis et des opportunités actuels pour la réalisation des ODD et la réduction des inégalités du sud. Cette analyse permet de dégager quelques propositions axées sur I'utilisation des nouvelles technologies de nature exponentielle en tant qu'outil clé et innovant pour atteindre ces objectifs, en fonction de leur capacité à accroître les impacts à des coûts réduits; en même temps, elles permettent de réduire l'écart technologique Nord-Sud, qui retarde le développement mondial et, par conséquent, la possibilité de se conformer aux ODD.

L'hypothèse qui a guidé les travaux est liée à la nécessité d'apporter des réponses innovantes et de promouvoir différents partenariats et projets de coopération qui impliquent diverses modalités et acteurs du Système International dans la réalisation des ODD et la réduction des inégalités. Enfin, sur la base de l'analyse effectuée, le travail propose la mise en œuvre d'un type de coopération internationale horizontale basée sur l'utilisation de technologies exponentielles pour surmonter les 169 objectifs fixés dans les 17 ODD et de réduire les inégalités, qu'il suggère $d^{\prime}$ appeler: coopération exponentielle (CoEx).

Mots clé: Objectives de développement durable (ODD), technologies exponentielles, coopération exponentielle (CoEx). 


\section{Introducción}

Las cuestiones vinculadas a las definiciones y los desafíos que plantea el desarrollo no son nuevas en la disciplina de las relaciones internacionales, ni en las actividades implementadas en la práctica tendientes a alcanzar los objetivos deseados. En este sentido, a partir de la Conferencia de Bandung de 1955, luego de la independencia de las excolonias asiáticas y africanas, las cuestiones vinculadas con el desarrollo pasaron a ser debatidas y tenidas en consideración por el Sistema Internacional (Lechini, 2009).

La posición desventajosa en términos de desarrollo relativo de los países del Sur y los desafíos para superar diversos obstáculos propiciaron, principalmente durante la década del setenta, grandes reflexiones y debates académicos sobre la materia, así como también diversas conferencias a nivel multilateral y de las diferentes regiones sobre las acciones tendientes a superar los desafíos del desarrollo y el papel de la cooperación internacional tradicional, la Sur-Sur y la Triangular en dicho escenario.

Sin embargo, y a pesar de cierto voluntarismo de los países del Sur, estos esfuerzos no tuvieron el efecto esperado en las décadas subsiguientes (Malacalza, 2009). Solamente a inicios del siglo XXI las cuestiones vinculadas con el desarrollo volvieron a ocupar el centro de las agendas gubernamentales; destacándose en este aspecto la Cooperación Sur-Sur (CSS) y Triangular en América Latina (AL) con el apoyo de la Secretaría General Iberoamericana (SEGIB).

Si bien la reemergencia de la CSS permitió experimentar grandes avances vinculados con el desarrollo y la reducción de desigualdades durante la denominada década dorada (de 2003 a 2013), quedaron muchos desafíos pendientes; al tiempo que la misma se encuentra actualmente en una "etapa de incertidumbre" en el marco de una coyuntura económica y política inestable, la cual plantea un escenario de mayores desafíos para la región (Ayllón, 2015: 159).

A su vez, a nivel multilateral y en el marco de la Agenda de la Eficacia de la Ayuda fueron aprobados en 2015 los 17 Objetivos de Desarrollo Sostenible (ODS). Éstos sumaron nuevos objetivos a los antiguos Objetivos de Desarrollo del Milenio $(O D M)$ e introdujeron un enfoque de sostenibilidad a la agenda del desarrollo; así como también un enfoque de universalidad por medio del lema "nadie será dejado atrás". Para la consecución y medición de avances de estos objetivos, fueron acordadas metas e indicadores específicos adaptados a cada país que cuentan con el compromiso de la comunidad internacional para ser alcanzados en 2030. 
A partir de su aprobación, los ODS se transformaron en "[...] una guía de orientación para la Cl, incluyendo obviamente a la CSS" (Surasky, 2017: 6). En este sentido, los países de AL que -según los Informes de la SEGIB (2016)-comparten la característica de todos llevar adelante actividades de CSS, deberían utilizar esa fortaleza (de vínculos regionales y experiencia en acciones de cooperación orientadas al desarrollo) como una herramienta clave sobre la cual consolidar las actividades y acciones tendientes a alcanzar los ODS.

No obstante, en función de los cambios políticos y económicos mencionados anteriormente, se alerta sobre la capacidad de mantener la unión regional en los debates sobre la gobernanza global y en las asociaciones regionales, a fin de superar los problemas que plantea el desarrollo; en dónde la CSS estaba jugando un papel muy relevante. Por lo que se destaca que, independientemente de las orientaciones políticas y la situación económica, mantener las asociaciones regionales que continúen potenciando las acciones de CSS y Triangular en favor de la superación de los desafíos que plantea el desarrollo sostenible y la consecución de los ODS; resulta fundamental.

En función de la referida coyuntura y de la realidad que continúa evidenciando cada vez más la necesidad de seguir trabajando y cooperando en favor del desarrollo (ahora en clave de sostenibilidad), el presente trabajo se cuestiona acerca de las posibles acciones a ser llevadas adelante por los países, en particular los latinoamericanos y sobre el papel que pueden jugar las nuevas tecnologías como herramientas para superar los ODS y disminuir las desigualdades. Identificándose la necesidad de continuar cooperando entre los países del Sur y proponiéndose la implementación de la Cooperación Exponencial (CoEx) entendida como una modalidad clave de apoyo y aceleración de las tres principales áreas del desarrollo sostenible (social, ambiental y económica), en la medida que es intensiva en tecnología exponencial e incorpora a todos los actores del Sistema, destacando a Estados, empresas, filantropía, ONGs y sociedad civil organizada.

\section{Actualidad regional frente a los desafíos del desarrollo sostenible}

A pesar del crecimiento de $\mathrm{AL}$ en la última década, la región continúa siendo la más desigual del mundo, con una estructura productiva y exportadora basada en ventajas comparativas asociadas a su dotación de recursos naturales. En consecuencia, la región precisa superar las brechas estructurales que la limitan a fin 
de aumentar la productividad y la competitividad, fortaleciendo las instituciones y superando su rezago en innovación, en ciencia y tecnología (CEPAL, 2014). Esta desigualdad, ya no solo a nivel regional sino global, plantea un gran desafío para la consecución de los ODS puesto que la misma torna al crecimiento y al desarrollo no sostenibles; por lo que se entiende que un trabajo orientado al desarrollo integral debe, primeramente, atacary superar las causas del subdesarrolloy la desigualdad.

Si bien el crecimiento económico y las iniciativas políticas de integración regional en AL han sido fundamentales y grandes dinamizadoras de las actividades de CSS para el desarrollo durante la década dorada; en función de la paulatina desaceleración del período actual, aparece el cuestionamiento acerca de si la CSS va a mantenerse consolidada y trabajando orientada hacia la superación de esas brechas estructurales y reducción de las desigualdades, contribuyendo con el camino en la consecución de los ODS.

En este sentido, se identifican dos dificultades principales en función del cambio de coyuntura; por un lado, una desaceleración de la economía regional que hace más difícil justificar a la interna de los gobiernos la orientación de fondos y capacidades nacionales hacia actividades de CSS regional y, por otro, ciertos cambios en las estrategias de política exterior de los países de AL que, en algunos casos, puedan generar una cierta separación regional a partir de la no coincidencia de ideas y visiones de desarrollo entre los Estados.

En relación con estos desafíos se destaca la necesidad de mantener la unión regional, en primer lugar, para continuar trabajando en la profundización de los avances logrados en materia de CSS y, en segundo, a fin de mantener una posición unificada del Sur en los ámbitos multilaterales que le permitan a la región mayores posibilidades negociadoras en los temas vinculados con la gobernanza global, el desarrollo y las condiciones de acceso. Lo cual requiere

[...] que los gobernantes comprendan que a pesar de las diferentes percepciones de la política exterior no hay diferencias profundas entre ellos, porque comparten un lugar desventajoso en el sistema económico internacional (Ojeda, 2016: 177).

Por estos motivos e intentando superar la dependencia de ciclos económicos asociados al precio internacional de los commodities, se propone la posibilidad de utilización de la transferencia de tecnologías y estímulos a la innovación como respuesta clave ante los desafíos del desarrollo sostenible, en el entendido de que la misma se relaciona directamente con las causas estructurales del (sub)desarrollo. 


\section{I. La tecnología como herramienta para el desarrollo sostenible}

El aumento en los últimos años de la participación de los países emergentes en la cooperación internacional en el ámbito multilateral, la ha convertido en un "campo de batalla" en el que países del Norte y del Sur estarían redefiniendo los nuevos límites de la cooperación y del desarrollo internacional (Esteves y Assunção, 2014: 1787). Ello permite visualizar un margen de negociación para incorporar al debate sobre la Eficacia de la Ayuda a los temas vinculados con las nuevas tecnologías, haciendo énfasis en las que tengan capacidad de impactar positivamente al desarrollo sostenible.

El foco de la discusión hacia las acciones de cooperación intensivas en tecnología para superar los nuevos desafíos del desarrollo se relaciona con el argumento de que en la medida que se plantean objetivos más ambiciosos, como lo son los ODS en comparación con los ODM, las respuestas también deben serlo. Destacándose aquí la meta 17.6 de los ODS que hace referencia a la necesidad de:

mejorar la cooperación regional e internacional Norte-Sur, Sur-Sury Triangular en materia de ciencia, tecnología e innovación y el acceso a estas, y aumentar el intercambio de conocimientos en condiciones mutuamente convenidas, incluso mejorando la coordinación entre los mecanismos existentes, en particular a nivel de las Naciones Unidas, y mediante un mecanismo mundial de facilitación de la tecnología.

Atendiendo a ello, en 2019 y a pedido del Secretario General de las Naciones Unidas, Antonio Guterres, fue conformado un panel especial sobre cooperación digital, como primer paso en esta dirección, el cual trajo una serie de recomendaciones en favor del estímulo y mejora del acceso a la tecnología e innovación a nivel global desde una perspectiva de interdependencia y con el espíritu de maximizar los impactos positivos de las tecnologías digitales y minimizar sus daños en el área social, legal, ética y económica (ONU, 2019: 4).

En este sentido, se entiende que buena parte de las oportunidades para alcanzar los ODS se encuentra en la implementación de actividades intensivas en aprendizaje e innovación, en concordancia con la eficiencia schumpeteriana trabajada por la CEPAL; destacándose las potencialidades de la CSS y Triangular para alcanzar estos objetivos, en la medida que las mismas sean ampliadas (en actores) y diversificadas (en sus formas) a fin de garantizar el éxito de los objetivos de desarrollo sostenible en la región.

Si bien el trabajo en el marco de la Agenda Post-2015 realizó importantes reflexiones acerca de la necesidad de profundizar los objetivos para el desarrollo, 
adaptar las metas al territorio y ajustarlas a las necesidades locales; no obstante se observa que, en muchos casos y por diferentes motivos, las acciones planteadas para enfrentarlos acaban siendo similares. Por ello, si bien se destaca que muchos avances han sido logrados en cuanto a los niveles de desarrollo y acceso a bienes y servicios, las condiciones e indicadores de desigualdad, principalmente en $\mathrm{AL}$, hacen que todavía se esté lejos de alcanzar los niveles deseados, permaneciendo muchos desafíos pendientes.

En este sentido, se entiende que resultará muy difícil alcanzar los ODS con las mismas estrategias que fueron implementadas para intentar alcanzar los ODM que, a pesar de grandes avances, no lograron cumplir con diversas metas establecidas para 2015. La siguiente frase, atribuida a Albert Einstein, resume claramente el argumento que aquí se está intentando plantear: "The significant problems we have, cannot be solved at the same level of consciousness with which we created them". Por lo que se plantea la necesidad de trabajar sobre nuevas estrategias intensivas en un tipo particular de tecnología: la exponencial.

\section{Tecnología exponencial: consideraciones, oportunidades y desafíos}

En el Foro Político de Alto Nivel de las Naciones Unidas (HLPF, por sus siglas en inglés) de 2018 su Secretaria General, Amina Mohamed destacó que, si bien los países están mostrando ciertas evidencias de progreso en algunas áreas de los ODS, los mismos están siendo demasiado lentos e incluso, en algunos casos, se está retrocediendo. En función de ello, se destaca la necesidad de dar respuestas innovadoras capaces de brindar un avance de tipo exponencial en los diferentes indicadores del desarrollo. Puesto que, la magnitud de los desafíos establecidos en los ODS hace pensar acerca de la necesidad de implementar acciones efectivas, que maximicen sus efectos y a costos reducidos. No solo por el mero cumplimiento de una batería de metas e indicadores acordados nivel global, sino fundamentalmente porque es humana y medioambientalmente imperativo.

Por lo anterior, se entiende que la utilización de la tecnología exponencial para estos fines puede acercarnos al desafío en menor tiempo; debido a que la misma cumple con la doble condición de tener su crecimiento duplicado a cada año y/o su costo reducido a la mitad también anualmente. Por lo cual, si consciente y consistentemente se utilizasen las herramientas que la robótica, las TICs, la nano y biotecnología, la realidad virtual y la inteligencia artificial proporcionan para 
fines relacionados con el acceso, el desarrollo y la sostenibilidad, se entiende que se estaría frente a un universo con mayores oportunidades de alcanzar los desafíos planteados en los ODS.

Adicionalmente, debe destacarse que la tecnología es neutra por naturaleza y que el impacto que las mismas puedan generar dependerá de las decisiones en relación con su aplicación, así como de su reglamentación y acuerdos entre sectores. En este sentido, frente al avance innegable de estas tecnologías, se plantea la necesidad de colocar incentivos en su impacto positivo y neutralización de posibles problemas para que, en vez de ser vistas como amenaza, las mismas puedan comenzar a ser abordadas por las teorías sociales como una oportunidad de avance para los objetivos orientados al desarrollo, la reducción de la pobreza y la sostenibilidad medioambiental, en la medida que sean orientados al bien común.

El presente trabajo entiende que, los avances tecnológicos con capacidad de impactar positivamente en el desarrollo humano y medioambiental deben estar orientados y puestos al servicio de la comunidad y no solamente destinados al lucro y crecimiento empresarial. Abonando a este punto, se destacan los postulados de la economista Mariana Mazzucato (2018), quien destaca que, a diferencia de los que muchos piensan, las principales innovaciones tecnológicas no han surgido dentro de empresas de manera aislada; sino que han sido posibles gracias al apoyo de fondos gubernamentales de estímulo a aprendizaje e investigación en ciencia y tecnología. Lo que representa un motivo más para justificar que las mismas sean compartidas con toda la sociedad y en beneficio de las diversas comunidades.

En este sentido, pueden encontrarse en la actualidad variadas evidencias empíricas de estas nuevas tecnologías desarrolladas e implementadas para la mejora en los sistemas de salud, en la prevención y detección de enfermedades; así como en construcciones habitacionales (a costos reducidos) post catástrofes naturales, asistencia humanitaria y cuidados medio ambientales, entre otros. La adaptación de dichas tecnologías a la realidad y necesidades de cada país, sobre la base de los principios de la CSS y Triangular, en asociación con los gobiernos, las redes regionales, el sector empresarial, la sociedad civil y la academia junto con los conocimientos de los cuales ya se dispone en la región; se entiende que podría ser una valiosa herramienta en favor del desarrollo humano y la consecución de los ODS.

Adicionalmente, la utilización de nuevas tecnologías de carácter exponencial para alcanzar los ODS aumenta las probabilidades de éxito, puesto que multiplica las capacidades y efectos; a la vez que reduce los tiempos y suele disminuir los costos de producción y/o ejecución. Dichas características son consideradas fundamentales 
para países como los latinoamericanos, ya que los mismos cuentan con capacidades y fondos limitados para estos fines. Asimismo, por el hecho de maximizar los impactos a menores costos, permite la democratización del acceso a determinados bienes y servicios básicos, hoy no garantizados por algunos gobiernos; a la vez que, propicia un avance de índole global en concordancia con el principio de inclusión de la Agenda 2030 que pretende "no dejar a nadie atrás".

Por tanto, si la utilización de la tecnología exponencial aparece como herramienta clave para superar los desafíos que plantea el desarrollo sostenible y contribuir en la superación de desigualdades; la cooperación internacional vinculada a estos temas debería ser la herramienta por excelencia a ser utilizada como agente multiplicador y democratizador de este tipo de tecnologías. El presente trabajo, propone denominar a estas actividades de cooperación internacional intensivas en nuevas tecnologías de la información, robótica, nano y biotecnología, realidad virtual e inteligencia artificial, entre otras como Cooperación Exponencial (CoEx).

En síntesis, frente a los cambios inevitables ante los cuales la sociedad internacional se encuentra actualmente, resulta determinante que las respuestas dadas por los gobiernos para enfrentar los retos presentes y futuros vinculados al desarrollo sostenible sean por lo menos regionales -sino globales-e innovadoras en sus formas y modalidades; al tiempo que deben ser intensivas en tecnología e involucrar a todos los actores del sistema. Entendiendo que, frente a problemas y desafíos de carácter global, las soluciones efectivas y sostenibles en el tiempo no serán encontradas en acciones aisladas, sino que a partir de la coordinación y cooperación internacional.

\section{I. Oportunidades y evidencias en favor del desarrollo sostenible}

La realidad actual demuestra que no nos encontramos más en una era de cambios, sino en un cambio de era; marcado por lo que el Foro Económico de Davos denominó de cuarta revolución industrial, impulsada por las tecnologías digitales y de la información. Éstas, si utilizadas de forma consciente y sistemática, tienen la capacidad de impactar transversalmente de forma positiva a toda la Agenda de ODS. En este sentido y sin ánimo de realizar un análisis exhaustivo, pues las evidencias de avance son diversas, se presentan aquí algunos ejemplos que sirven como indicadores de mejora en diversas áreas, sobre las cuales es necesario continuar trabajando para el cumplimiento de las metas en 2030. 
Adicionalmente se destaca que debido a que varios de los ODS se superponen, en muchos casos, al impactar positivamente en uno de ellos, consecuentemente se afecta de manera indirecta en el avance de otros de los objetivos, sus respectivas metas e indicadores asociados. No obstante, esta superposición conlleva también a una alerta sobre el manejo y control de las denominadas externalidades negativas.

Entre las herramientas tecnológicas que pueden ser colocadas al servicio de los ODS se destaca la importancia de la Big data. Por medio de la misma, es posible proveer todos los datos e indicadores necesarios para acompañar y comparar los avances de objetivos, metas e indicadores por países, ciudades y también entre las corporaciones. Ello permitirá una mejor comprensión y comunicación sobre la situación actual de cada uno de los indicadores, así como la clara identificación de avances y retrocesos; al tiempo que facilitará la definición de prioridades de acción que se encuentren alineadas con el principio que busca no dejar a nadie atrás. En definitiva, permite el acceso a la información de calidad para comunicar, concientizar y tomar acciones de forma asertiva y más eficiente.

Otro desarrollo tecnológico que está permitiendo grandes avances en diversos ODS se relaciona con las impresoras 3D, las cuales están siendo usadas en diversas áreas. A modo de ejemplo se destaca la Organización sin fines de lucro New Story ${ }^{2}$ que está actualmente imprimiendo casas para ayudar en la reconstrucción de villas y ciudades post desastres naturales, así como también de poblaciones excluidas sin acceso a vivienda.

Adicionalmente, se destaca que estas impresoras 3D también están siendo utilizadas como aliadas de la bio y nanotecnología en la impresión de órganos para trasplantes, así como también en la impresión de prótesis. Esta biotecnología también se encuentra trabajando y avanzando fuertemente en la detección temprana de ciertas enfermedades y la prevención de pandemias, a fin de disminuir los indicadores de muertes evitables.

Si bien resta mucho trabajo y perfeccionamiento en estas áreas, estos avances evidencian la capacidad exponencial de superar problemas mundiales como la falta de vivienda, mejorar la calidad de vida por medio de prótesis e implantes; así como también prevenir muertes evitables por medio de trasplantes y la prevención y detección temprana de diferentes enfermedades. No obstante, debe alertarse aquí sobre la necesidad de una regulación apropiada que permita el acceso global

${ }^{2}$ Más información en: https://newstorycharity.org/about/ 
a estos avances, restrinja y controle es uso de los datos personales y evite ciertos lobbies de las grandes corporaciones.

En los desafíos relacionados con el hambre y la mejora en la nutrición, grandes avances están siendo presentados en la producción agroindustrial. Algunos ejemplos se relacionan con las plantaciones verticales en ciudades que, además de aumentar y acelerar la producción, a su vez disminuye la contaminación generada por el transporte de los productos y reduce su precio, al acortar las cadenas de valor. La agricultura de precisión aparece como otro ejemplo relevante que, por medio de la utilización de las tecnologías de navegación por satélite y machine learning, entre otras, permite estimar las variaciones, siembras y fertilizaciones óptimas que mejoren el rendimiento de los cultivos. Al tiempo que también se está avanzando en alternativas para disminuir el consumo de carne y el impacto medioambiental ocasionado por los gases de efecto invernadero y utilización de agua, a través de la sustitución del consumo de la carne animal por proteína vegetal, como es el caso de la empresa Beyond Meat3.

Aquí, además de la necesidad de continuar avanzando, debe alertarse sobre la importancia de análisis en relación con los beneficios nutricionales de estas alternativas, el control del uso de agrotóxicos, colorantes y aditivos; así como también las garantías de acceso y el establecimiento de un precio justo, a fin de contribuir con la consecución del ODS 2: hambre cero, sin por ello impactar negativamente en otras metas de ODS como la calidad de la nutrición, la contaminación o depredación ambiental e inclusive las condiciones de trabajo y derechos humanos.

Otro ejemplo, en este caso aplicado en el área social, se relaciona con la utilización de lentes de realidad virtual con la intención de colocar a las personas en la realidad de otra, a fin de contribuir en la generación de empatía y la toma de decisiones más acertadas. Un ejemplo de ello es la utilización de estos lentes para experimentar la vida en un campo de refugiados o en determinadas situaciones de exclusión. La utilización de drones como asistentes humanitarios para el transporte y la entrega de paquetes de primeros auxilios, agua y comida a lugares de difícil o imposible acceso en un determinado momento crítico, también aparece como otro ejemplo cada vez más utilizado.

Por su parte, la utilización del blockchain está permitiendo la inclusión financiera y el acceso al crédito para las poblaciones más vulnerables y jugando un papel relevante en la inclusión financiera y acceso al crédito. A modo de ejemplo a inicios

${ }^{3}$ Más información en: https://beyondmeat.com/about 
de 2018, las Naciones Unidas utilizaron esta tecnología con refugiados sirios para garantizar su acceso a determinados bienes y servicios básicos.

Obviamente, resulta fundamental destacar aquí la importancia de la inteligencia artificial en los avances de las nuevas tecnologías de tipo exponencial y su impacto en diversas áreas que viabilizan mejoras de extrema importancia en el desarrollo de nuestras actividades laborales, de producción y consumo; al tiempo que también resulta relevante alertar sobre su impacto en nuestra autonomía, privacidad y formas de trabajos, entre otros.

Finalmente y con el fin de garantizar la escalabilidad del conocimiento, las innovaciones y las buenas prácticas, debe destacarse como punto primordial la construcción de la infraestructura necesaria que garantice la conectividad y el acceso, junto con los sistemas nacionales de capacitación y formación en programación, ciencia y tecnología; así como también la reglamentación que garantice su democratización y utilización apropiada.

\subsection{Desafíos para la región}

En la medida que los avances tecnológicos son una realidad instalada, se plantea la necesidad de reflexionar acerca de algunos desafíos vinculados con las garantías de acceso; así como también la democratización de los mismos y su utilización en favor del desarrollo y la reducción de las desigualdades.

En este sentido, ya el último informe Obama de la Casa Blanca de diciembre de 2016 denominado "Artificial Intelligence, Automation, and the Economy" 4 trataba de estos asuntos y destacaba la importancia de utilizar las nuevas tecnologías y la inteligencia artificial en favor del desarrollo sostenible, puntualizando también en la necesidad de implementar todas las reglamentaciones necesarias para que las mismas puedan ser utilizadas de manera segura:

Advances in Artificial Intelligence (Al) technology and related fields have opened up new markets and new opportunities for progress in critical areas such as health, education, energy, economic inclusion, social welfare, and the environment (2016:3). [... ] economic incentives and public policy can play a significant role in shaping the direction and effects of technological change (2016: 9).

${ }^{4}$ Informe disponible en: https://obamawhitehouse.archives.gov/sites/whitehouse.gov/files/documents/ Artificial-Intelligence-Automation-Economy.PDF 
Debe ser destacada aquí la importancia de la regulación gubernamental, alertándose sobre la necesidad de trabajar en favor de una reglamentación proactiva (no reactiva) por parte de los gobiernos latinoamericanos, debido a que actualmente la innovación avanza a mayor velocidad que la reglamentación a su respecto. Ello requiere de atención en dos sentidos, por un lado, debido a que el aumento de esa tecnología en la medida que las misma no sea compartida, aumenta las desigualdades que se están intentando superar con el plateo y, por el otro, en función de que, si no es creada una reglamentación apropiada para su regulación, dicha tecnología puede ser utilizada para fines que nada tengan que ver con el desarrollo o incluso que puedan volverse en su contra.

Por lo anterior, la cooperación internacional y la construcción de un acuerdo global (o al menos inicialmente regional) en este sentido también resulta altamente relevante, a fin de orientar la aplicación de estas nuevas tecnologías para fines vinculados con el desarrollo sostenible en diversas áreas y no en detrimento de éste, ni en la ampliación de desigualdades y/o brechas tecnológicas.

Otro desafío importante se relaciona con la infraestructura de telecomunicaciones necesaria para garantizar la conectividad y el aprovechamiento de estos beneficios, tanto de la ciudadanía como del sistema empresarial. Permitiendo el acceso a un servicio de calidad y a un costo accesible. Por lo que las garantías de acceso, es otro desafío importante para considerar en este apartado. En este sentido, para el caso latinoamericano se destaca que el acceso a la banda ancha es principalmente móvil. De acuerdo con la Unión Internacional de Telecomunicaciones (2017) en 2016 , un $51,3 \%$ de los habitantes latinoamericanos contaba con acceso a telefonía móvil; mientras que solamente el $8,94 \%$ contaba con acceso a internet fijo.

Si bien aún queda un gran trabajo por la frente en cuanto a la inclusión y al cierre de la brecha digital en la región, se destaca que el acceso aumenta anualmente, al tiempo que la tecnología móvil también avanza encontrándose actualmente en la quinta generación (5G), lo cual requiere de mayores inversiones en infraestructura para acompañar el mencionado crecimiento. A partir de la inclusión y el aumento exponencial de los datos en la red, aparece otro gran desafío vinculado con la ciberseguridad, la privacidad y el control de datos en las redes. Pues el respeto de los derechos y confidencialidad de los usuarios debe ser garantizado.

De todos los desafíos planteados, se entiende que el debate en torno de la Gobernanza de internet y las nuevas tecnologías es uno de los retos más importantes ante los cuales el mundo y, consecuentemente, la región latinoamericana se encuentra actualmente. El mismo, será el encargado de democratizar el 
acceso e impactar verdaderamente en la reducción de las brechas tecnológicas que limitan las posibilidades de superar los desafíos planteados por los ODS y reducir las desigualdades.

\section{La importancia de la Gobernanza de las nuevas tecnologías}

A pesar del trabajo que realiza la Escuela del Sur de Gobernanza de Internet, al observar las dinámicas actuales se identifica que la gobernanza de la tecnología se está dando en el marco de los acuerdos económico-comerciales y no en el marco de foros políticos-multilaterales, lo que limita ampliamente la capacidad de acceso de países en desarrollo como los latinoamericanos, al tiempo que favorece a las grandes corporaciones en detrimento del bienestar social global; a pesar de que, como destacado anteriormente, las grandes innovaciones tecnológicas han sido posibles gracias al financiamiento estatal en ciencia y tecnología.

Adicionalmente, si se analizan los datos relacionados al registro de patentes para el caso latinoamericano, de acuerdo con la CEPAL con base en la información estadística de la Organización Mundial de la Propiedad Intelectual (OMPI), la participación mundial de AL en la solicitud de patentes desde 1990 no ha logrado superar el $5 \%$; lo que refuerza la necesidad de que $\mathrm{AL}$ participe activa y efectivamente de la cuarta revolución industrial.

Esta brecha tecnológica persistente entre el Norte y el Sur hace necesario continuar la discusión sobre el sistema de patentes, si se pretende alcanzar un desarrollo global sostenible. Recordando, a su vez, la oportunidad actual que el "campo de batalla" de discusión sobre los conceptos y límites de la CID destacado por Esteves y Assunção (2014), brinda actualmente. Motivo por el cual se destaca aquí la importancia de incorporar al debate la consideración de ciertos avances tecnológicos, en particular los que cuenten con potencial para superar desafíos orientados al desarrollo sostenible, como bienes públicos globales. Ello permitirá la participación de los Estados en las discusiones sobre el uso de las tecnologías, la orientación, propósito y distribución de las mismas.

Asimismo, además de la opción de considerar a las tecnologías que contribuyan con el desarrollo sostenible como bienes públicos, existe otra alternativa que podrían explorar los países de AL a través del

[... ] establecimiento de un fondo que compre y libere patentes relevantes desde la perspectiva de la sostenibilidad. Esa reducción de los costos de adquisición de tecnología 
puede tener un efecto mayor si opera en un mercado regional integrado, que posibilite el aprovechamiento de economías de escala (CEPAL, 2016: 152).

El punto que se intenta levantar en este apartado se relaciona con la consolidación de nuevas formas de dependencia que precisan ser abordadas, debatidas y superadas. Destacándose la importancia para el Sur de continuar trabajando en los ámbitos multilaterales en favor de la disminución de la brecha tecnológica, puesto que la misma será la gran dinamizadora y aceleradora del desarrollo sostenible y posibilitadora de la reducción o aumento de la exclusión y desigualdad.

\section{El rol de los diversos actores en favor del desarrollo sostenible}

La realidad actual que desafía tanto al planeta como a las formas de vida y desarrollo humano, hacen necesario repensar las concepciones y actividades relacionadas con ese desarrollo. En este sentido, los ODS expresan los diversos desafíos a ser superados a nivel global y también a nivel de cada país. La consecución de estos objetivos solo será posible si además de repensar las acciones sostenibles a ser implementadas, las mismas se ejecuten con mayor intensidad e incluyendo a todos los actores gubernamentales y no gubernamentales. Entendiendo por actor a los

[...] sujetos colectivos o individuales que posean una identidad, que les permita reconocerse como tales, con capacidad de acción estratégica y recursos para su realización (Acuña y Chudnovsky, 2013: 36).

Las referidas acciones en favor del desarrollo sostenible deberán entonces ser compartidas y potencializadas a través de la cooperación internacional, la cual precisa ser acordada entre los diferentes Estados y/o unidades subnacionales a fin de estar en concordancia con los planes y estrategias de desarrollo nacional. A su vez, también se hace precisa la incorporación y asociación con los demás actores no gubernamentales en el marco de una alianza global en favor de desarrollo que maximice las posibilidades y acelere los resultados. En este sentido se destaca la importancia de la participación del sector privado, la sociedad civil organizada y la academia como agentes de cambio y desarrollo con la capacidad de impactar positiva y transversalmente en toda la agenda de ODS.

Con relación a la acción de los privados y la filantropía en las actividades vinculadas con el desarrollo sostenible se destaca un aumento en su interés y participación desde hace algunos años. En este sentido, en el año 2000 fue lanzado el Pacto Global de las Naciones Unidas como la mayor iniciativa de sostenibilidad corporativa 
voluntaria. El mismo crece anualmente $y$, si bien es necesaria continuar sumando empresas y esfuerzos en favor de estos objetivos, en la actualidad reúne a más de 12.000 empresas en 170 países con el objetivo de alinear las actividades de negocio a los diez principios del Pacto, relacionados con los derechos humanos, el cumplimiento de normas laborales y la protección medioambiental, entre otros ${ }^{5}$.

La necesidad de mayores fondos y acciones en favor de los ODS, así como una comprensión más sistémica de estas cuestiones, ha otorgado más espacio al sector privado dentro de la agenda del desarrollo sostenible; lo cual favorecerá el cumplimiento de los objetivos, en la medida que dichas acciones sean realizadas a partir de una mayor y mejor coordinación con los Gobiernos, a fin de garantizar que las mismas se encuentren alineadas con las agendas o planes nacionales para el desarrollo. Al tiempo que también, se destaca que las actividades intensivas en conocimiento, transferencia de nuevas tecnologías e instalación de capacidades debe buscar y estimular la apropiación por parte de los países en detrimento de una estrategia de dependencia comercial.

Por su parte, la Sociedad Civil cada vez más organizada se encuentra jugando un papel de relevancia en la concientización, así como también en los reclamos y pedidos de informaciones acerca de la sostenibilidad de las actividades y procedimientos llevados adelante tanto de sus gobiernos nacionales y departamentales, así como también de las corporaciones y empresas que operan local y globalmente. En este marco, diversos grupos sociales y también particulares se han organizado en los últimos años para promover y llevar adelante acciones vinculadas con el desarrollo sostenible.

Dentro de estos colectivos, los jóvenes han empezado a ganar especial destaque en función de diversos aspectos; por un lado, su educación está comenzando a tomar en consideración e incorporar estas cuestiones, estimulando una comprensión y sensibilidad mayor hacia la necesidad de repensar los modelos actuales de desarrollo; al tiempo que también serán estas generaciones más jóvenes las que sufrirán -en algunos casos- y se beneficiarán -en otros- de los mayores impactos medioambientales a causa de los efectos del calentamiento global y de los cambios en el sistema educativo y del mundo del trabajo, a partir de la incorporación de las nuevas tecnologías. Motivos por los cuales se destaca aquí el papel fundamental que las nuevas generaciones tienen, en la toma de conciencia e implementación de acciones en favor del desarrollo sostenible, la inclusión y la reducción de desigualdades.

${ }^{5}$ Más información disponible en: www.unglobalcompact.org 
En este contexto se destaca el papel del Organismo Internacional de Juventud para Iberoamérica (OIJ), debido a que es la única institución internacional dedicada exclusivamente a la juventud, la cual articula la cooperación internacional en esta materia y representa a los 21 países que la integran, en las acciones y políticas necesarias a ser llevadas a cabo en materia de juventud. El OIJ destaca por su importante trabajo de estímulo y visibilidad de la juventud como agentes de cambio y transformación social, contribuyendo para una lberoamérica más inclusiva, solidaria, unida y pacífica; destacando sus valores vinculados con la igualdad de género, el principio de no discriminación, el valor de la diversidad y la igualdad generacionalt.

Este tipo de acciones resultan de fundamental importancia para la inclusión de las nuevas y futuras generaciones al debate del desarrollo sostenible, de la educación, del mundo del trabajo y para garantizar su participación efectiva en los procesos de decisiones políticas en esta materia; debido a que, en última instancia, serán los más impactados por los nuevos cambios sociales y medioambientales. En este sentido, son justamente estas nuevas generaciones las cuales se encuentran, en mayor medida, proponiendo nuevas ideas y creando soluciones apoyadas en la tecnología exponencial en favor del desarrollo sostenible.

Finalmente, se destaca el papel que la academia desarrolla en favor del desarrollo sostenible, pues, además de ser la encargada de la formación cada vez más intensiva en ciencia, tecnología y programación, entre otros; es en su marco donde muchas de las innovaciones y avances tienen lugar, así como también es la responsable por las reflexiones sobre los efectos que estas nuevas tecnologías pueden tener a largo plazo, más allá del objetivo inicial para el cual fueron desarrolladas, a partir de estudios multivariables de difusión e impacto.

\section{La Cooperación Exponencial (CoEx) como propuesta es- tratégica para la consecución de los ODS}

En función de las oportunidades y beneficios evidenciados a partir de la utilización de la tecnología exponencial para el desarrollo sostenible y a la disminución de las desigualdades para no dejar a nadie atrás, se destaca la importancia de expandir este conocimiento y las buenas prácticas por medio de la cooperación internacional

${ }^{6}$ Más información en: https://oij.org 
especialmente con los países del Sur y particularmente con $\mathrm{AL}$-por ser la región más desigual del mundo-; destacando para ello a las modalidades horizontales de CSS y Triangular. Sobre esta cooperación horizontal, Lechini destaca que:

La llamada cooperación horizontal entre estados de similar desarrollo refiere en la práctica a la cooperación técnica y científico-tecnológica. La cooperación técnica alude a la transferencia de capacidades técnicas y administrativas (el know-how o saber-hacer) entre los propios países en desarrollo, en tanto la segunda se orienta a la realización de actividades conjuntas de investigación para promover avances científicos y tecnológicos que les permitan un desarrollo integral [... En esa línea se considera la llamada 'cooperación en cascada', pues los países relativamente más desarrollados -que han adaptado las tecnologías de los países industrializados a sus propios territorios y necesidades- las transfieren a sus socios del Sur, tal como lo hizo Brasil con su 'tecnología tropicalizada' (Lechini, 2009:68).

Por otro lado, además de las acciones Estatales, se destaca también la posibilidad de asociación con el sector privado a fin de crear sinergias público-privadas y estimular la apropiación de las referidas innovaciones a nivel de los países. Por lo que:

No se trata en este caso solamente de las posibles alianzas entre Estados del Sur o con terceras partes para trabajar bajo estructuras de CSS o Cooperación Triangular (CT) sino de crear espacios y dinámicas de trabajo conjunto que reúnan al Estado con actores no estatales, sean capaces de aprovechar las ventajas que cada parte puede aportar al logro de objetivos comunes, contribuyan a lograr el apalancamiento de recursos e identifiquen y refuercen las sinergias existentes (Surasky, 2017: 4).

Como visto anteriormente, la acción del sector privado en esta área se encuentra en aumento desde hace algunos años, por lo que se entiende que su participación debe ser considerada como una variable adicional para potenciar las acciones orientadas al desarrollo; en la medida que dichas acciones se encuentren alineadas con las agendas o planes nacionales para el desarrollo sostenible y que, a su vez, estimulen la apropiación por parte de los países.

Por su parte, las actividades de Cooperación Triangular (CT) también se encuentran en progresivo aumento en la región. De acuerdo con el Informe de CSS y Triangular que realiza la SEGIB, se destaca que las iniciativas de CT Iberoamericanas entre 2006 y 2014 fueron multiplicadas por nueve (SEGIB, 2016: 135). En este sentido, desde dicha Organización en el marco del Seminario de CSS con miras a los ODS -organizado junto con la CEPAL-, se destaca:

[...] el papel relevante que ha adoptado la cooperación triangular como parte de esta nueva arquitectura de la cooperación internacional para el desarrollo. Este tipo de cooperación, ya instalada en América Latina y el Caribe, permite el aprovechamiento de las ventajas comparativas que cada socio involucrado (tradicional, emergente y beneficiario) pueda aportar en virtud de sus características propias, genera beneficios compartidos, 
propicia la replicabilidad de los proyectos de cooperación y, a la vez, es efectiva para el cumplimiento de los objetivos de Desarrollo Sostenible (PIFCSS, 2016: 16).

Esta $\mathrm{CT}$, en su versión más tradicional, permitiría que un socio con una colaboración de capital no demasiado significativa pueda generar un efecto exponencial relevante en la creación, utilización y/o aplicación de estas tecnologías para el desarrollo; así como también, pueden explorarse las actividades con países y/o corporaciones que ya posean tecnologías avanzadas validadas y en ejecución para que las mismas puedan ser adaptada para ser transferidas y aplicadas entre los países del Sur a fin de superar desafíos de desarrollo sostenible.

Paralelamente, también existe la opción de asociaciones estratégicas con otros países del Sur global, como es el caso de India o de China (destacados por la creación y desarrollo de nuevas tecnologías), los cuales pueden configurarse como aliados claves de $\mathrm{AL}$ en la transferencia, adaptación e implementación de las nuevas tecnologías orientadas hacia la solución de problemas de desarrollo y superación de los ODS.

Todas estas modalidades de cooperación de tipo horizontal, si realizadas de forma intensiva en materia de capacitación y transferencia de tecnología, se entiende que son excelentes estrategias de respuesta a los nuevos desafíos del desarrollo; puesto que posibilitan la ejecución de proyectos asertivos, enfocados en las necesidades locales, con alto grado de impacto y con efectos exponenciales gracias a la introducción de la tecnología.

Por tanto, se plantea aquí una respuesta alternativa e innovadora, enmarcada en un tipo de cooperación horizontal no tradicional, que este trabajo propone en denominar "Cooperación Exponencial (CoEx)", en el entendido de que la misma es intensiva en la transferencia de tecnología exponencial, la cual multiplica las capacidades, maximiza los impactos y reduce los costos; potencializando las posibilidades de alcanzar los ODS, puesto que estos avances tecnológicos tienen la capacidad de ser utilizados de manera transversal a toda la Agenda 2030.

Este tipo de cooperación internacional horizontal intensiva en intercambio y conocimiento tecnológico con capacidad de crecimiento exponencial -puesto que duplican su crecimiento anualmente y/o disminuye su costo a la mitad-, incluye la participación de diversos actores como Estados, unidades subnacionales, organizaciones de la sociedad civil, representaciones de diversos colectivos, empresas, academia, etc. que impactan transversalmente en la consecución de diversos objetivos y metas, simultáneamente. 
Finalmente, por lo expuesto anteriormente, se entiende que la cooperación horizontal para el desarrollo sostenible en la región deberá enfocarse especialmente, en los términos de Jesús (2007), en las dinámicas que involucren la articulación entre conocimiento-innovación-educación, lo que permitirá a Latinoamérica avanzar en el camino del desarrollo sostenible y la disminución de desigualdades a través de las nuevas tecnologías y la participación de todos los actores del sistema, por medio de la Cooperación Exponencial (CoEx).

\section{Conclusiones}

El presente trabajo pretendió realizar un aporte a la reflexión acerca de los nuevos desafíos que plantean los ODS y sobre cómo la tecnología y la cooperación internacional pueden aportar en este sentido.

Para ello se realizó un análisis sobre la actualidad latinoamericana, destacándose la necesidad de mantener las asociaciones regionales que continúen potenciando las acciones de CSS y Triangular en favor de la superación de los desafíos que plantea el desarrollo, la consecución de los ODSy la reducción de las desigualdades.

Paralelamente, por medio del análisis, se identificó la necesidad de brindar respuestas novedosas e innovadoras para afrontar desafíos globales de gran magnitud a nivel social y medioambiental. A partir de dicha constatación, se identificó a los avances tecnológicos como los grandes dinamizadores del desarrollo sostenible, en la medida que el acuerdo de objetivos más ambiciosos requiere también de acciones más creativas. Por lo cual, se entiende que la utilización de la tecnología exponencial puede acercarnos al desafío, debido a que cumple con la condición de tener su crecimiento duplicado a cada año y/o su costo es reducido a la mitad anualmente.

En función de ello, se identificó la necesidad de que AL disminuya la actual brecha tecnológica en relación con los países centrales; para lo cual el trabajo propuso dos alternativas relacionadas con la gobernanza tecnológica, que tienen que ver con: la posibilidad de considerar ciertos avances tecnológicos (que tengan el potencial de impactar positivamente en el desarrollo sostenible) como bienes globales, y/o la creación de fondos globales que compren y liberen las patentes de ese tipo de avances mencionados.

A su vez, se trabajó sobre la importancia de incluir en estas actividades de cooperación a las asociaciones entre los actores gubernamentales y no gubernamentales, 
destacando al sector empresarial, la sociedad civil organizada, la juventud y la academia, como agentes dinamizadores del desarrollo y con la capacidad de impactar transversalmente en la agenda de los ODS.

En relación con la implementación de las referidas tecnologías, se trabajó sobre el papel fundamental de la cooperación internacional horizontal entre países en desarrollo intensiva en tecnología exponencial, con capacidad de impactar positivamente en el desarrollo sostenible y la reducción de las desigualdades.

Finalmente, sobre esta cooperación se planteó una respuesta alternativa e innovadora enmarcada en un tipo de cooperación horizontal no tradicional e intensiva en tecnología, que este trabajo propone llamar de Cooperación Exponencial (CoEx). Este tipo de cooperación internacional horizontal intensiva en conocimiento e intercambio tecnológico con capacidad de crecimiento exponencial, puesto que duplica su crecimiento anualmente y/o disminuye su costo a la mitad; incluye la participación de diversos actores como Estados, empresas, academia, organizaciones de la sociedad civil y representaciones de diversos colectivos que impactan transversalmente en la reducción de desigualdades y en la consecución de diversos objetivos y metas sostenibles, simultáneamente.

\section{Referencias}

ACUÑA, C. y ChudnOvsky, M. (2013), "Cómo entender las instituciones y su relación con la política: lo bueno, lo malo y lo feo de las instituciones y los institucionalismos", en AcuÑA, C. (comp.), ¿ Cuánto importan las instituciones? Gobierno, Estado y actores en la política argentina, Siglo Veintiuno, Buenos Aires, pp. 13-67.

Ayıón, B. (2015), "La Cooperación Sur-Sur en América Latina y Caribe. De una época dorada a una fase incierta", en CRIES "América Latina y el Caribe y el nuevo sistema internacional: Miradas desde el Sur", Anuario de la Integración Regional de América Latina y el Caribe, $\mathrm{n}^{\circ} 11$. Coordinadora Regional de Investigaciones Económicas y Sociales, pp. 134-170.

BeLI, L. y CaVALI, O. (Coords.) (2018), Gobernanza y regulaciones de Internet en América Latina. Análisis sobre infraestructura, privacidad, ciberseguridad y evoluciones tecnológicas en honor de los diez años de la South School on Internet Governance, FGV Direito Rio. 
CEPAL (2014), "Cambio estructural para la igualdad. Una visión integrada del desarrollo", Comisión Económica para América Latina-Naciones Unidas, Santiago de Chile, LC/G.2604.

- (2016), "Horizontes 2030: la igualdad en el centro del desarrollo sostenible", Comisión Económica para América Latina-Naciones Unidas, Santiago de Chile, LC/G.2660.

Esteves, P. y Assunç̃̃̃, M. (2014), "South-South Cooperation and the International Battlefield: Between the OECD and the UN", en Third World Quarterly, vol. 35, $\mathrm{n}^{\circ} 10$, pp. 1775-1790.

JESÚs, S. (2007), "Conocimiento, cooperación y desarrollo", Revista lberoamericana de Ciencia, Tecnología y Sociedad - CTS, vol. 3, n 8, pp. 195-208.

LECHINI, G. (2009), "La cooperación Sur-Sury la búsqueda de autonomía en América Latina ¿Mito o Realidad?", Revista Relaciones Internacionales, n 12, GERI - UA Madrid, España, pp. 55-81.

MALCALZA, B. (2009), Viejos y nuevos debates en torno a la ayuda internacional al desarrollo, Mirada4, FLACSO, Argentina, pp. 85-110.

Mazzucato, M. (2018), The value of everything: Making and Taking in the Global Economy, Allen Lane.

OJEDA, T. (2016), Relaciones internacionales y cooperación con enfoque Sur-Sur, Libros de la Catarata, Madrid.

ONU (2019), Report of the UN Secretary-General's High-level Panel on Digital Cooperation "The Age of digital interdependence".

ONU Mujeres (2018), Informe de seguimiento de los ODS "Transformar las promesas en acción: la igualdad de género en la Agenda 2030".

PIFCSS - Programa Iberoamericano para el Fortalecimiento de la Cooperación Sur-Sur (2016), Iberoamérica frente al desafío de los Objetivos de Desarrollo Sostenible. Una mirada desde la Cooperación Sur-Sur, Seminario Internacional, CEPAL, Santiago de Chile.

SEGIB (2016), Informe de la Cooperación Sur-Sur en Iberoamérica, Secretaría General Iberoamericana, Madrid.

SURASKY, J. (2017), Cooperación Sur-Sur en la Agenda 2030 y hacia el PABA+40, Instituto de Relaciones Internacionales, Universidad Nacional de La Plata. 